\title{
Can my robotic home cleaner be happy? Issues about emotional expression in non-bio-inspired robots.
}

\author{
Andrea Bonarini ${ }^{1}$
}

\begin{abstract}
In many robotic applications a robot body should have a functional shape that cannot include bio-inspired elements, but it would still be important that the robot can express emotions, moods, or a character, to make it acceptable, and to involve its users. Dynamic signals from movement can be exploited to provide this expression, while the robot is acting to perform its task. A research effort has been started to find general emotion expression models for actions that could be applied to any kind of robot to obtain believable and easily detectable emotional expressions. On his path, the need for a unified representation of emotional expression emerged. A framework to define action characteristics that could be used to represent emotions is proposed in this paper. Guidelines are provided to identify quantitative models and numerical values for parameters, which can be used to design and engineer emotional robot actions. A set of robots having different shapes, movement possibilities, and goals have been implemented following these guidelines. Thanks to the proposed framework, different models to implement emotional expression could now be compared in a sound way. The question mentioned in the title can now be answered in a justified way.
\end{abstract}

\section{Keywords}

Emotional robot, Emotion, Emotional movement, Autonomous robots, Human-Robot interaction, Social robot

\section{Introduction}

In many robotic applications the robot body should have a functional shape that do not include bio-inspired elements. Let's call these robots as "NOBIBots". Examples are vacuum cleaners, flying drones, lawn movers, but also smart phones, automatic doors, and cars. In all these cases, it is not possible to exploit bio-inspired features like face or arms to express emotion or a character. However, these robots have to interact with people, and emotional expression may promote an interaction more interesting than that commonly exploited with simple appliances, thus improving acceptance, effective interaction, and market opportunities. Moreover, in co-working or collaborative tasks the immediate appreciation of what is going on is important to achieve common tasks, and set up an effective relationship between people and robots. Emotion expression is part of this setting, too.

The amount of works studying non-bio-inspired features to express emotions in robotics (e.g., (Novikova and Watts, 2015)) is small in comparison to those that use human-like features.

Emotional expressions can be obtained in these cases by a careful design of actions, intended as producers of emotional signals, too. Since NOBIBots have to perform their tasks, the emotional expression should not, in principle, affect too much their performance.

Inspired by Mori's conjectures about uncanny valley (Mori, 1970), we can say that actions have a primary role in expressing emotion, even more than static posture, as it comes also from many experiments on emotion recognition in humans (e.g., (Atkinson, Dittrich, Gemmell, and Young, 2004), (Dittrich, Troscianko, Lea, and Morgan, 1996)). In particular, we assume that, for NOBIBots, static postures are mostly inexpressive, and motion can satisfactorily express emotions only if it can exploit the range of values needed for each feature, with specific reference to each robot base exterior characteristic. In particular,

\footnotetext{
${ }^{1} \mathrm{Al}$ and Robotics Lab, Dipartimento di Elettronica, Informazione e Bioingegneria, Politecnico di Milano, Italy
}

Corresponding author:

Andrea Bonarini

DEIB-Politecnico di Milano

Piazza Leonardo da Vinci, 32

20133 Milano - Italy.

Email: andrea.bonarini@polimi.it 
experiments where the human body is hidden, and only characteristic points associated to moving body parts are shown (e.g., point light movies, (Atkinson et al., 2004), (Dittrich et al., 1996)) support the fact that the possibility to perceive explicitly face or limbs is not needed to obtain high emotion recognition rates.

This paper presents a general framework for emotion expression through movement, which could be applied to any kind of robot to obtain believable and highly detectable emotion expressions. The framework is inspired by models developed for similar needs in other fields such as acting, dance, mime, puppetry, and cartoon animation. Basing upon the qualitative models there defined, and discussed in Section 3), elements of the framework have been defined and guidelines can be provided to identify quantitative models and numerical values for parameters, which can be used to design and engineer actions for robots. The proposed models consider features such as speed, acceleration, rhythm, but also body dimensions, and expectations coming from perceived body weight or past experience about movement. In many situations, failing to consider even only one of the critical aspects makes the emotion expression less recognizable, according to the "perceptual magnet effect" (Kuhl, 1991), in which stimuli close to a category boundary are judged by observers to be more dissimilar than stimuli that are away from a category boundary. In this case, emotional expression may lose its effect, or may even bring to emotional discomfort (Moore, 2012).

In the following, the term "emotional cue" is intended to denote sets of features that can be detected and interpreted as expression of both emotions, i.e. reactions to some event, moods, i.e. states that can be induced by a combination of events and mental states and that persist for a longer time, and character traits, i.e. stable psychological states, characteristic of an individual, possibly modulated by moods and emotions. A review about the differences among these terms can be found in (Ekkekakis, 2012). While these phenomena share similar emotional expressions, the interaction among them is certainly interesting, but not in the scope of this paper, focused instead on emotional expressions in NOBIbots, through movement.

People perceive activity as emotionally expressive when it matches their own experience, as discussed in section 2 .

The modelling approaches defined in entertainment arts, from which inspiration has been taken to define the framework presented in this paper, are discussed in sections 3.
In the framework, some action features are considered as possible elements that can add to "functional" actions (i.e., those devised to achieve the task) some emotional characteristics; they are presented in section 4 .

In section 5 the framework for emotional model representation is introduced together with guidelines to identify its parameters.

The proposed framework has been derived from the implementation of a set of robots having different shapes, movement possibilities, and goals, some of which are presented in Section 6. As discussed there, some of the emotions usually considered in literature can be relatively easy to render also with few degrees of freedom in the robot body, while others require more elements and a wider range of action possibilities.

Thanks to the presented framework and guidelines, the paper's title question will be answered at the last section, in a justified way.

\section{Emotion in people and animals}

Emotions in people are deemed to be both innate and socially learned mechanisms functional to live a better life. Emotions are related to physiological changes occurring when some specific external event happens (Cacioppo, Tassinary, and Berntson, 2000). These physiological changes may reflect on (or induce) movements and other perceivable signals (e.g., face colouring), which might be interpreted as emotional cues.

Emotional cue interpretation has always been a personally developed ability, and became more structured only with psychophysiological studies (e.g., (Cacioppo et al., 2000; Ekman, 1999)).

Trials aimed at investigating how untrained people (as the users of generic a robot would be) could recognize emotional cues from moving subjects put in evidence how only very few features of the human movement can be considered as universally valid for this task (Kikhia et al., 2014; Mentis and Johansson, 2013). In particular, speed and acceleration turned out to be relevant features, but also relative movements of specific body parts, in particular trunk, head, and arms. These are considered in the framework.

By transfer, people tend to attribute to some animals the ability to express emotions with signals similar to those used by humans. Of course, animals with bodies similar to the human one may have similar means to produce acts that can be interpreted as emotional cues (Darwin, 1998), but we easily attribute at least some emotional expressions also to dolphins, horses, or geese, not mentioning the more traditional pets (e.g. (Bloom and Friedman, 2013)). 
The attribution of emotional expressions to animals with bodies dissimilar from human ones can give us some hints about what people could consider as emotional cues, which could be adopted also for NOBIbots. For instance, we may say that a dolphin is happy when it moves parts of the body with a ample and fast oscillatory rhythm, which is possible when staying on its tail outside water, impossible with the proper rhythm while swimming underwater. So, a dolphin can be perceived as happy only out of the water. Even there, it is less easy to say that it is sad, because it cannot show the characteristic features of this emotion, e.g., by bending the body and moving slowly. The meaning and possible values of the terms in italic are discussed in section 4 .

Many models to classify emotions in people have been proposed (see commented reviews in (Marsella, Gratch, and Petta, 2010; Scherer, 2010; Kowalczuk and Czubenko, 2016)). In this paper, reference is done, when needed, to the circumplex model (Russell, 1980), as this is the most commonly considered reference in Human-Robot Interaction; in particular it is considered the version where emotions are classified according to only two dimensions, represented on Cartesian axes, namely Valence and Arousal. Valence indicates how much the emotion is pleasant and ranges from negative to positive, while Arousal indicates how much energy is involved in emotion.

\section{Emotion in entertainment arts}

Emotion representation plays a relevant role in entertainment arts. Most of the knowledge to do this is still orally transmitted, since training of performers is generally done live, in a multi-modal context, including movements, postures, voice, body percussion and any other exploitation of communication channels. Some models have been defined, but they are often qualitative, and they assume that shared knowledge could support the understanding of undefined terms. In arts where people is not directly performing, but a mechanical implementation of actions is needed (such as in graphical animation), some basic elements have been defined more precisely.

In performing arts, the performer tends to reproduce real life, including emotional expression. This is done by actors mainly by imitation, possibly entering in the emotional state to reproduce, by recalling a past experience (Stanislavski, 1989), or making the experience in a socially reacting context (Lecoq, 2000; Johnstone, 2012). In this area, emotional expression is not precisely coded, and its implementation relies on shared experience, assumed as common to both actors, directors, and the public. From this area we can only draw the suggestion to make emotional expression as related as possible to what people is used at. Again, this experience can be considered at least in part innate, and in part socially developed, so that the emotional expression can be at least in part dependent on the social environment: different societies may perceive the intended emotional expression in different ways. This is a relevant issue, but it is related to the content of the model, not to its structure, so it is not considered in this paper.

In dance, body expression through action is essential to performance, so models for it have been developed, mostly when it emerged the need to transmit the artistic design of the choreographer, over the personal expression of the dancer. In the middle of the last century, some choreographers started to define models of dance movement (e.g., (Laban and Ullmann, 1968; Loman and Merman, 1996)), that lead to the definition of movement models and notations to represent choreographies. Roboticists, as other professionals, have got interest in these models, since they provide a quite structured way to identify at least some characteristics of movement, although none of these characteristics is originally described in engineering terms, directly usable for designing robot movements. In particular, a part of the Laban's model is related to expressivity, and considers features like: direction of movement with respect to a target (direct-indirect), flow (boundfree), attitude towards time, or speed, (sustainedquick), weight (heavy-light). These features have been used to frame emotional expression (Camurri, Lagerlöf, and Volpe, 2003), and considered in different experiments to design robot movements to express emotions(e.g., (Lourens, Van Berkel, and Barakova, 2010; Masuda and Kato, 2010; Knight and Simmons, 2014; Barakova and Lourens, 2010)). In some cases, Laban-formed actors performed the movements corresponding to emotion expression, and these were used as basis to develop analogous movements for robots, unfortunately affected by specific limitations related to motor speed, shape, locomotion media, etc. In other cases, they have been used both to select dimensions of investigation (e.g. (Angel Fernandez, 2016; Barakova and Lourens, 2010)) and to design movements (e.g. (Knight and Simmons, 2014; Barakova and Lourens, 2010)).

In theatrical arts (acting, dancing, and also puppeteering) there is a strong accent on the body movement w.r.t. face expression, in particular on movements perceivable by the audience from distance. The body can be seen as composed by three main sections: the head, the triangle shoulders-abdomen 
(chest), and the lower part (pelvis and legs), plus the arms. The relative movements and positions of these segments can produce emotion expression. In puppeteering, sometimes the emotional expression is limited to opening-closing gestures, possibly integrated with forward-backward bending, and sometimes lateral bending, often sufficient to render a sufficiently wide range of emotions, to complement in a coherent way speech or sound. The tradition of Commedia dell'Arte is considered in many schools as a basis for actor's formation; here, characters and mood are often associated to combinations of the same kind of postures and movements of the three segments, which produce immediate and reliable feeling in the audience.

Not all robots have these three segments, but an increasing number of social robots are including at least one articulated joint (e.g. Care-O-bot (Kittmann et al., 2015), Pepper (Aldebaran, 2016), Furby (Hasbro, 2016)) or a possibility to deform the body (e.g. KeepOn (Kozima, Michalowski, and Nakagawa, 2009) and Tofu (Wistort, 2010)) just for emotion expression. It turns out that the possibility to express bending, turning, and openness is extremely relevant to produce recognizable emotion expressions (e.g., (Angel and Bonarini, 2014; Angel Fernandez, 2016)).

Another important source of inspiration is cartoon animation. From the beginning, cartoon characters were designed to render emotions with movement. In many cases, animal or human shapes were used for characters, but the possibility to have animated objects was exploited from the very beginning (Thomas and Johnston, 1995): the half-full flour sack was explored among the first objects to understand how to design movement to render emotional situations. Most objects had a character or at least conveyed an emotional status; let's consider, for example, the brooms in Fantasia, or the butler-clock in The Beauty and the Beast, or even Wall-E and Eve, the robots, or the iconic Pixar's Luxo lamp by Lasseter (Lasseter, 1987). Animators tend to exaggerate movements, and body deformations, to make the emotional status more evident.

Due to the medium they have to work with, animators developed an interesting way to represent movement: they have to state where are all the parts of a moving object in every single frame of a film. Frames are presented at a fixed frame rate (originally, 24 frames/sec): this gives hints about speed and acceleration we may have in cartoons, which we may mimic in live scenes with robots. With this approach, it is natural to define trajectories in terms of positions of the object components in the subsequent frames. Modifications of a trajectory can be easily implemented by drawing the object in one or another place, by remembering that the physiological and cognitive elaboration of subsequent images has to interpolate images of 'close enough' object's poses to reconstruct the movement as a continuous displacement instead than just as appearing and disappearing. Nowadays, the typical way of designing movements in animation consists of defining key frames and leave to computer programs the burden to generate the needed frames between key frames, by applying given laws (e.g., movements and deformations can be generated to follow parametric splines). Among the entertainment arts, animation is the one exploiting computers, thus requiring a formal definition of everything. As described in section 5 , key poses for the robot actuators can be identified analogously to key frames, and control laws can be designed to implement appropriate trajectories, by considering both the robot features and the quality of movement.

\section{Emotion expression features}

In this paper, only kinematic features are considered to express emotions. They are introduced with reference to displacement, but the extension of the basic concepts to other communication channels (e.g., sound, light) that could be used to express emotion is possible. In this section, the considered features are introduced and their values discussed, in the next section we will see how it would be possible to build a model upon them.

People tend to classify the values of features as belonging to individually (and often unconsciously) defined, fuzzy classes. In the following, examples of these classes for the different features are introduced together with their relationships to everyday experience. They can be adapted to contextual features such as the dimension of the body, or the physical interaction with specific people. Only examples of models are presented, derived from the robots presented in Section 6. The framework provides a conceptual frame that has to be instantiated case by case. Thanks to the conceptual frame, different models can be compared by considering not only vague terms, as present in the currently available literature, but their grounding on actual values used in robots. This might put in evidence in a sound way the basic mechanisms that could be exploited to express emotions.

We provide descriptions of value classes in terms of fuzzy sets (Zadeh, 1965), a very general tool, proved as appropriate to define such type of classifications done by humans. A fuzzy set is a set (of values) for which a membership function is defined: a value can belong 
to a fuzzy set with a given degree of membership from 0 to 1 . This is a tool to represent the people's way of defining classes in everyday life, in a robust and effective way. The membership functions can have any shape: triangular, trapezoidal, bell-shaped, and even single valued spikes (singletons) or rectangular, the last corresponding to crisp intervals. The definition of the membership functions is part of the modelling process, and a discussion of the different choices is not in the scope of this paper.

A fuzzy set is identified by a label, usually a linguistic term, whose meaning is formally defined by the corresponding membership function.

In the rest of this section, the features considered in this work are listed, and examples of fuzzy sets are presented; most of them can be defined with respect to a reference value, on which the 'Regular' fuzzy set is defined. One of the interesting features of this modelling approach is that the reference value can be moved without changing the meaning and shapes of the other fuzzy sets. When possible, this makes it possible to re-use emotion expression models on different robots, just by defining the feature considered as 'Regular', since all the rest adjusts on that.

Fuzzy sets like the ones used in these models can be identified by showing to people the robots actually moving in the operating context, and asking how they would classify the different robot's feature values (e.g., values of speed) that they are observing.

\subsection{Displacement}

Let us consider the movement of a rigid body in 2-D, e.g., a robot base moving on the floor. According to the background reported in sections 2 and 3, three basic features are considered: the Direction of movement w.r.t. the interacting agent, the Speed of robot, and its Acceleration.

4.1.1 Direction. In human-robot interaction, it is relevant to model the direction of movement as relative to the person the robot is interacting with, since this provides a strong characterization of the act. For instance, moving fast against a person may be interpreted as an aggressive behaviour, while moving at the same speed in the opposite direction can be interpreted as a scary get-away. Direction classes relevant for emotion expression may be labeled as: 'Towards', 'ToLeft', 'ToRight', 'Away', or with a finergraded classification, if this is needed to characterize different emotional expressions. For the examples reported later on, these values are enough. In Figure 1.a is reported a, typically circular, distribution of fuzzy sets defined over the direction depicted in Figure 1.b. a.

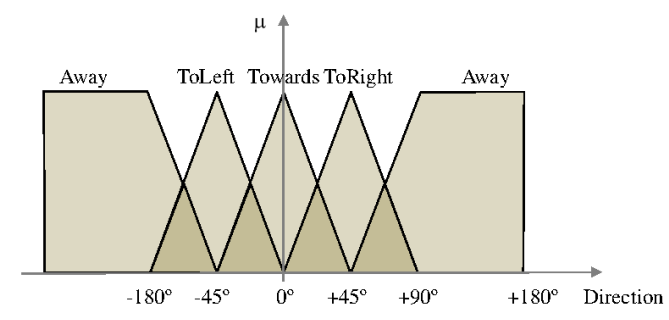

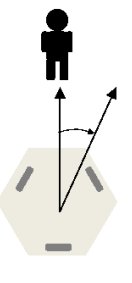

b.
Figure 1. A possible fuzzy set definition for the feature Direction, a typical, circular variable (a). The convention for Direction (b).

4.1.2 Translational speed. When interacting with other biological agents, we expect that robots can move at a speed ranging on values comparable to what people and interacting animals can afford for interaction. Typically, in close interaction, speed can be in a range from 0 to about $1.5 \mathrm{~m} / \mathrm{sec}$, and, actually, this is more or less the range of speed for social robots on the market. We can consider to classify translational speed as 'Still', 'Slow', 'Regular', and 'Fast', with reference to what is usually called as 'Walk'. So, we may have a slow walk, a regular walk, a fast walk, and so on. Faster speed is considered as 'Run', and it is not in the scope of this paper, since here we focus on close interaction.

In general, a robot has a typical operation speed (e.g., about $0.4 \mathrm{~m} / \mathrm{sec}$ for a home cleaner), which may be considered as a reference alternative to average human walking speed (around $1.3 \mathrm{~m} / \mathrm{sec}$ ). This opens the possibility for a context-related definition of the reference speed, so the actual value of the top point of the 'Regular' fuzzy set. The perceived 'Regular' speed may also vary according to the dimension of the robot: a $20 \mathrm{~cm}$ long robot can be considered to show a 'Regular' speed when running at $0.4 \mathrm{~m} / \mathrm{sec}$, while and autonomous car may run at different 'Regular' speed according to the context, e.g. down-town the 'Regular' speed could be $14 \mathrm{~m} / \mathrm{sec}$, while on a speedway could be $30 \mathrm{~m} / \mathrm{sec}$. Given that we are assuming to produce emotional expression by modulating speed, in the models we can represent a percent difference w.r.t. the 'Regular' speed to characterize terms such as 'Slow' or 'Fast'. We assume that, once identified the expected 'Regular' speed with reference to context, we can define a model to express emotions based on labelled percent increments or decrements of it.

An example of a distribution of fuzzy sets for Translational Speed is shown in Figure 2. Notice that, for this robot, the 'Fast' speed definition is not covering values higher than $150 \%$ of the 'Regular' speed, since speed cannot go over that. Usually, we have constraints 


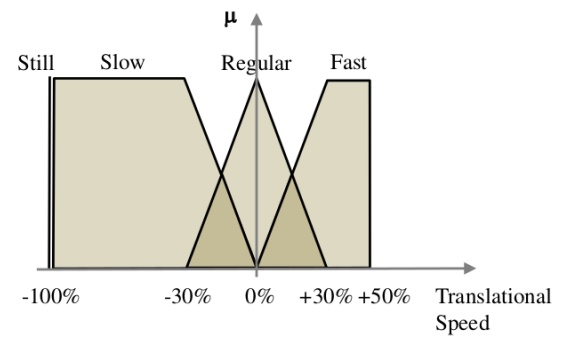

Figure 2. A possible fuzzy set definition for the feature Translational Speed. For instance, a percentage speed of $-0.21 \%$ is classified as 'Slow' with a degree 0.7 , and as 'Regular' with a degree 0.3.

on the moving possibilities, which may suggest different distributions, and may affect emotional expression, as discussed in section 5.3.

4.1.3 Rotational speed. People are used at quite high rotational speeds, in the order of up to 300 degree/sec, easily possible for humans when they roll over. As a reference, a robotic home cleaner may roll over at about 100 degree/sec. For instance, let us consider 'NoRotation', 'SlowRotation', 'RegularRotation', and 'FastRotation', both 'Clockwise' and 'Counter-clockwise'. Also in the case of rotational speed we might consider to have a reference rotational speed depending on the robot and the context, but the emotional expression for this dimension is quite independent from the specific base, and the general considerations done in Section 5 apply on the absolute rotational speed values. Since, in common interaction, rotation is usually not continuous, but actuated for a limited angle, acceleration is important for this feature.

4.1.4 Acceleration. Acceleration, both for translation and rotation is one of the most effective features in emotion expression. By managing acceleration, a movement can start and stop in different ways, and can vary its speed. It is related to the "weight" dimension in the Laban's model(e.g., (Lourens et al., 2010; Masuda and Kato, 2010; Knight and Simmons, 2014; Barakova and Lourens, 2010)). As an example, ranges of $\pm 1 \mathrm{~m} / \mathrm{sec}^{2}$ for translational acceleration, and of \pm 300 degrees $/$ sec $^{2}$ for rotational acceleration are considered. Specific cases for acceleration are related to 'Start' and 'Stop', for which labels like 'Sudden', 'Regular', 'Slow' can be defined. Acceleration during movement can be described by 'NoAcceleration' to keep the speed constant, 'SlowAcceleration', 'FastAcceleration', and the analogous for deceleration.

Depending on the selected kind of model representation, acceleration may be defined explicitly, with the mentioned terms and corresponding fuzzy sets, or indirectly, by providing velocity set points on a trajectory, and the corresponding time stamps, as it happens in the models derived from the animation tradition.

4.1.5 Rhythm. In our case, 'rhythm' is a regular, repeated pattern of movements. The musical attributes of rhythm may be translated for movement. The accent is a stress given to an element, with respect to its neighbours. It is used to make an element recognizable from others. In movement, the accent makes it possible to distinguish a specific movement from others. It can be implemented as a strong enough change in velocity, like, for instance, stop or start, or clearly perceivable acceleration/deceleration. The stronger the variation, the stronger is the accent. Each distinguishable element have a duration, like that of the notes in a musical score. For instance, the robot may start at a slow speed for 0.5 seconds, then stop for 0.3 seconds, and repeat this pattern for some time, to give the idea of sobbing; or it can slowly alternate left and right turns while slowly moving backward to show to be scared. With accent and duration it is possible to define expressive patterns. The third component of rhythm is tempo, i.e., how fast a pattern is executed. The same pattern, executed at different tempos may express different emotions, or even miss any meaningful expressive power.

4.1.6 Path. The above mentioned features characterize the movement along a path, which, in agreement with Laban's dimensions, can be designed to be either direct, such as a linear path from the current pose to the final one, or more or less indirect, such as a spline between the two poses. Moreover, it can constrain more or less the movement, so leaving to the robot degrees of freedom to be explored while aiming at the target.

\subsection{Movement of body elements}

Features analogous to the ones presented for displacement can be adopted for parts of the body that can move with respect to each other. These could be analogous to limbs, but also simple elements, or even the only top and bottom parts of the body, like, e.g. in Tofu (Wistort, 2010), and in Keepon (Kozima et al., 2009).

Bending the body is one of the most representative ways to show emotions, and it has been considered in many robots such as Furby (Hasbro, 2016), and Care-O-Bot (Parlitz, Hägele, Klein, Seifert, and Dautenhahn, 2008). For some emotions, bending plays even the role of most characteristic feature. For instance, as recognized from different backgrounds (e.g., (Thomas and Johnston, 1995; Ekman, 2004; 
Kleinsmith and Bianchi-Berthouze, 2013)), slowly bending forward is one of the main elements to express Sadness, while slightly bending to the back and maintaining the position gives an eager look, and the same, done fast and readily followed by a regular position gives the idea of Surprise, even without any staring eyes.

\section{Modelling emotion expression}

Given the motion feature characterizations presented in the previous section, it is possible to define different models to express emotions. Motion expression patterns can be defined as temporal distribution of differential poses on a path. Once a path is defined by the application, the emotion expression pattern can be applied to transform it in an emotional performance.

\subsection{Emotion expression patterns}

The emotion pattern representation is like a musical (or animation) score, where poses are like notes (or key frames), each described by a position on the path, possibly associated to a time stamp, a velocity, or an acceleration. We consider them as expressed using the terms associated to the fuzzy sets above mentioned. Like notes stay on different lines of the pentagram, poses on different actuators stay on different paths. Each pose should be distinguishable from each other, because of some feature value. The higher the difference, the more significant is the accent on that pose. This way to describe motion, as a time-stamped sequence of different feature values, is analogous to the one used to design movements for Sony Rolly (a very expressive NOBIBot (Sony, 2008)), and not far from Laban's notation used to represent movements in choreography (Laban and Ullmann, 1968). In both these cases, a direct reference with the musical score constrains the definition of timing. In more general applications, no relationship with music is exploited, so the design of emotional expression can define its own characteristic timing without any constraint.

\subsection{Adding emotion to a pre-defined action}

In many applications, the robot has to perform a task by moving to achieve a goal. Let's call these actions as task actions. Examples of goals are: "reach a given pose", and "stay at a given distance from a moving person". Usually, there are some degrees of freedom to perform the action, e.g., the pose should be reached, but the trajectory is free, or the time constraints to reach the pose are not strict. By considering the constraints, an emotional expression might be added to the task action, which is designed according to some optimality criterion, such as "reach the target speed as soon as possible", or "follow the ideal trajectory as close as possible". Notice that the apparently straightforward way of using only some channels to express emotion (e.g., light or sound) while leaving motion unaffected by emotion, may be perceived as a weird mismatch between communication channels (Moore, 2012). Therefore, emotional expression should affect also the task actions.

The functional movement for a robot is considered to have been generated by considering only the task of the robot, e.g., cleaning a room. A classical description of this movement consists of a path, described by functions in the variables' space (e.g., for planar movements, $p(x, y, \theta))$, and a timing law stating how the movement develops in time along the path $(s(t))$. A complex path may consist of several way points among which simple paths are defined. A path can be planned, by considering the starting and target poses, and constraints.

Emotional expression should be composed with this two-fold representation of movement by avoiding to disrupt it, but, at the same time aiming at being perceived. In principle, both the shape of the path and the timing law can be modified by superimposing the law needed to implement the emotional pattern.

A different kind of model, much closer to a designer's mindset, consists of key poses, possibly associated to time stamps, to annotate the functional movement description. In this framework, each emotion expression is implemented by a pattern of poses that describe the modifications of the functional trajectory. In section 3, experiments are reported where this modelling approach was adopted.

\subsection{Constraints}

A lot of, sometimes contradictory, suggestions about the selection of qualitative values for features that could be used to represent emotions have been presented in human emotion literature (e.g., (Kleinsmith and Bianchi-Berthouze, 2013; Crane and Gross, 2013)). Despite discrepancies, some principles may be easily accepted, for instance, happiness has to do with openness, high speed, free movement, while sadness is better expressed by closing postures, slow speed, small displacement.

Descriptions of feature values for emotional expression are usually given in linguistic terms. It is known that linguistic terms can be agreed upon more easily than precise, mathematical models (Zadeh, 1975), but 
to implement emotional expression on actual robots, a set of numerical values for features should be selected.

Basing on the fuzzy representation of these terms, it is possible to select the actual values for the motion features, for instance by picking the center of mass of the corresponding membership function.

However, the general model based on the fuzzy classification might need adjustments to match constraints of diverse nature. Like in any robotic application, there are constraints related to the actual possibilities of motors to bring the robot to the desired speed and acceleration (physical constraints), but also constraints deriving from expectations that people can have about the emotional expression (expression constraints). These latter may be of various nature. Many are related to physical coherence: for instance, we do not expect that a hundred kilograms robot would be able to show great acceleration, so its way to be happy, if any, would be possibly different from the one of a $20 \mathrm{~cm}$ long robot which might be able to exploit proper acceleration values. Moreover, values for speed and acceleration should be physically coherent with the visual aspect, upon which expectations of the interacting person is based on. An assistive robot weighting $200 \mathrm{~kg}$ and moving at a maximum speed of $50 \mathrm{~cm} / \mathrm{sec}$ would hardly be able to show happiness, because cannot show (nor it would be worth, given the operating environment) high enough acceleration to produce the fast alternate movements associated to happiness; an emotional expression based only on a smiling face on its screen would result in a mismatching communication (Moore, 2012), possibly accepted as an explicit signal to express an internal state, but hardly inducing a shared emotion.

Some other constraints come from the need to implement a difference in motion quality (difference constraints) compatible both with the functionality of the operations and the user's possibility to perceive it; for instance, a robot normally moving slowly, will probably not be able to show sadness by moving even more slowly. This might be the case of the assistive robot just mentioned, which might have a 'Regular' operation speed of $40 \mathrm{~cm} / \mathrm{sec}$ for safety reasons, and the difference with a 'Slow' speed, required by the Sadness model, would reduce the speed to $13 \mathrm{~cm} / \mathrm{sec}$, if we take the term definitions given in Figure 2, and the center of mass value of the fuzzy set is considered to compute the 'Slow' feature value. In this application, $13 \mathrm{~cm} / \mathrm{sec}$ would be definitely too low for effective operation, and the fuzzyness of the proposed model comes to support. The percentage decrement in the general emotion model is given as a fuzzy set. If this turns out to be too low (or too high) to be implemented, or too close to the neutral one (so not perceivable as anything different from neutral action) the value to be adopted to generate the final feature value can be moved within the fuzzy set, in quest of an acceptable value. If the fuzzy set has a flat top, as in this case (see Figure 2), a more appropriate value (e.g., $20 \mathrm{~cm} / \mathrm{sec}$ ) can be selected in the flat top interval without risking to reduce emotion interpretation, since in this case the fuzzy set has that validated shape, and all the values on the flat top interval are conceptually equivalent by construction. Otherwise, when considering values in the slope part of the fuzzy membership function, the emotion designer has to decide whether to leave the model to try to achieve the final goal; in this case, some information about the expected level of achievement of the emotion recognition may come from the fuzzy set involved to produce such variation. For instance, in our case we might safely decide to accept a reduction of speed up to $70 \%$ (flat top interval), while if we go for a speed reduction of, e.g. $45 \%$ we know that the possibility that this is interpreted as Slow (as required by the Sadness definition) is reduced by $50 \%$.

\subsection{Summary}

In summary, the modelling process flows through the steps listed here below.

- Reference values identification: reference values and range for motion features (e.g., Speed) are identified.

- Label identification and fuzzy sets definition: for each variable involved in emotion expression the fuzzy classification of values in the range is defined.

- Emotion pattern definition: emotion expression patterns are defined by using the terms defined at the previous step. They could in principle be re-used.

- Emotion pattern application: the emotion patterns are applied on the non-emotional movement.

\section{Emotional robots}

In this section a few applications of the modelling framework are presented, with the aim of showing its representational power, and the various situations it enables to face. Experimental results related to these applications are published or submitted elsewhere.

\subsection{Evaluation considerations}

In almost all the trials, a direct experience with the robot was proposed, since we strongly believe 
that the complete physical interaction is the only possible way to really get the feeling. A live experience includes sounds, real threats, confrontation with actual dimensions and speed, real proxemics, and many other factors that cannot be correctly explored by showing movies. Moreover, the final application can always be only with a real robot.

Emotion recognition depends on many personal factors, so that any statistics is affected by problems due to the fact that subjects are considered as valid samples for a population expected to be uniform; unfortunately, each person is different from each other for what concerns emotions, so no subject can be really considered as a good sample of such a varied population. Moreover, a lot of potentially relevant information is often neither collected, nor considered, thus making any statistical analysis even less significant. We share with (Machajdik and Hanbury, 2010) their critical analysis about emotion classification, in particular what concerns the arbitrary selection of emotional categories, which is not an easy task, as discussed in psychological studies (e.g., (Osgood, Suci, and Tannenbaum, 1957)) and many others concerning emotion classification (e.g., (Wang and Cheong, 2006) for emotion classification of movies). Many works focus on the six terms proposed by Ekman as basic emotions (Happiness, Surprise, Anger, Sadness, Fear and Disgust - (Ekman, 1999)), although some of them (e.g., Disgust), seem to require very specific facial features, not present in NOBIBots, to be reliably recognized. As it is clear from works done on emotion recognition with large numbers of subjects selected to be representative of a general population (e.g., IAPS for affective image classification (Lang, Bradley, and Cuthbert, 2008)), the values of features describing emotions distribute on quite wide ranges, and the corresponding clusters, only the center of which is often considered as reference, can cover intervals as wide as a quarter of the interval of values for each dimension (e.g., in the Arousal-Valence space), even excluding outliers. An interesting approach has been proposed in (Wang and Cheong, 2006) where affective classification of movies was not done in the ArousalValence space trying to identify directly the features' values, but considering many aspects that characterize movies, finding a relationship with emotional labels, and mapping these back to the Arousal-Valence space, coming to clusters somehow different from the ones originally associated to emotional labels. We independently followed a similar approach to classify robot actions from the features that it was possible to control, i.e., the ones we have included in our model.
The process of labelling an experience, such as looking at a robot that shows some emotional expression, is often affected by personal background and evaluation criteria. Moreover, terms used to name expressions are often imposed by the researchers in a context that is quite different from the one where the personal meaning for the same terms used to label that experience got formed. Therefore, it would be more significant to consider induced feelings and effective interaction (indirect evaluation) in place of direct labelling with an emotional term, as done, for instance, in (Lakatos et al., 2014).

In the reported examples, the evaluation of emotion recognition was done following the traditional approach, by asking people to label the experience and by statistical evaluation of the results. Although this has been done on quite large numbers of subjects (order of hundreds) for this kind of evaluations, we know that they are still largely not enough representative of a general population. Other times, indirect evaluation has been performed.

\subsection{Jedi Trainer: avoiding emotions}

The first example we would like to mention is a physically interactive robogame application (Jedi Trainer (Martinoia, Calandriello, and Bonarini, 2013)) where an autonomous flying drone had to challenge a Jedi apprentice (as Luke Skywalker in the first movie of the StarWars saga) by flying around her/him and shooting a laser blast. The player has to parry the blast with a light saber. This game, brought our attention on the importance of emotional interaction in robogames. One of the first problems to be faced was that of giving the drone a kind of cool attitude, like the one in the movie, despite the evident problems induced by the low-cost sensors and setting. In particular, a control system had to be devised, able to smooth all actions, and to avoid anything that could be perceived as dangerous in the real life by the player. So, the aim was to eliminate any action that could induce the impression that the drone was really aggressive with the player, such as abrupt changes of speed in the direction of the player. In a sense, this was done with the aim contrary to the one that guided all the subsequent activities: identify the emotional cues that might occur unexpectedly (e.g., due to poor image quality) and design a control system that could prevent their expression. The game was played by more than 70 people in a controlled trial (ad many others freely), almost all enjoying the game and entering, as desired, in a flow state (Csikszentmihalyi, 1997), fully engaged in the game experience. 


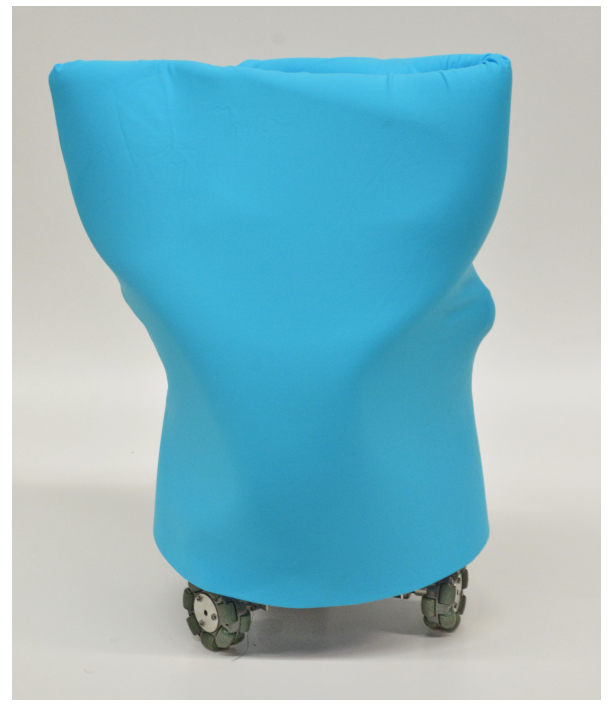

Figure 3. MARIOS, a holonomic base, with a neutral body.

If we had the framework at that time, we had identified the undesired emotional state in the user (Fear) and the corresponding undesired states in the drone (Aggressivity). Given the models of this last, in we had to move Speed away from a 'Fast' value associated to a direction 'Towards'. This was actually done, by tricking on the control program, where the solution is now hidden.

\subsection{Robot theatre: how difficult}

On the way to implement an autonomous robotic actor, the emotion expression of NOBIBot was studied. MARIOS (Mobile Autonomous Robot Interpreting On Stage - see Figure 3) is an holonomic robot, $40 \mathrm{~cm}$ wide and $60 \mathrm{~cm}$ high, able to run at up to $1.2 \mathrm{~m} / \mathrm{sec}$. Six case studies were performed (Angel Fernandez, 2016), while developing the modelling process that is now formalized in the proposed framework. The robot had to express the emotion along a straight path about 3 meters long. Emotional movements affected both the path and the timing law, and were represented as tangential and rotational speed targets on points of the reference trajectory. Although the base could in principle move also elements in its upper part, in these trials only the movement on the floor could be considered.

In the first case study, eight emotions were defined selecting feature values according to the designer's feelings. In particular, for Translational Speed these values were defined a priori: 'VerySlow' $(100 \mathrm{~mm} / \mathrm{s})$, 'Slow' (200 $\mathrm{mm} / \mathrm{s})$, 'RegularSlow $(300 \mathrm{~mm} / \mathrm{s})$ ', 'Regular' $(400 \mathrm{~mm} / \mathrm{s})$, and 'Fast' $(800 \mathrm{~mm} / \mathrm{s})$. For TangentialSpeed the values were:'None' $(0 \mathrm{~mm} / \mathrm{s})$,
'Slow' (300 mm/s), 'Medium' (500 $\mathrm{mm} / \mathrm{s})$, and 'Fast' $(800 \mathrm{~mm} / \mathrm{s})$. For BodyRotation (the maximum body rotation) the values were: 'None' (0 rad), 'Small' (0.1 rad), 'Medium' (0.3 rad), 'Large' (0.5 rad), and 'Huge' ( $0.7 \mathrm{rad})$. The emotions were implemented by considering these values:

Table 1. Features to implement emotional expression in MARIOS. First and second case study.

\begin{tabular}{|l|c|c|c|}
\hline Emotion & TSpeed & RSpeed & BodyRot \\
\hline Anger & Fast & Medium & Very Small \\
\hline Sadness & Very Slow & Slow & Small \\
\hline Fear & Normal Slow & None & None \\
\hline Embar. & Slow & Slow & Small \\
\hline Happiness & Fast & Fast & Small \\
\hline Disgust & Slow & None & Very Small \\
\hline Curiosity & Normal & Slow & Large \\
\hline Neutral & Normal & None & None \\
\hline
\end{tabular}

One emotional movement was showed to each subject, who was asked to select an emotion term in a list (Angel Fernandez, 2016). Only few emotions were recognized with a sufficiently high rate (Anger and Happiness). In a second case study a short scene to contextualize emotions was performed live (video available as https://www. youtube.com/watch ?v=AXAglJKLwbI). Again, only few emotions were reliably recognized (namely: Anger, Happiness, Fear, Curiosity) (Angel and Bonarini, 2014).

In the next case study subjects observed emotion expressions designed on emotion descriptions derived from Laban's models, as from table 2.

Table 2. Laban's features for the implemented emotions in the third case study, with relative references. The description of the features are the same as in the original sources.

\begin{tabular}{|c|l|}
\hline Emotion & Characteristics \\
\hline Anger & $\begin{array}{l}\text { Slow velocity, strong force and downward } \\
\text { body movement (Kleinsmith and Bianchi- } \\
\text { Berthouze, 2013) }\end{array}$ \\
\hline Happiness & $\begin{array}{l}\text { Sudden, hurried, fast, and strong } \\
\text { force (Crane and Gross, 2013) }\end{array}$ \\
\hline Sadness & $\begin{array}{l}\text { Low energy, slow, and tense (Kleinsmith and } \\
\text { Bianchi-Berthouze, 2013) }\end{array}$ \\
\hline Content & $\begin{array}{l}\text { Low energy (Kleinsmith and Bianchi- } \\
\text { Berthouze, 2013) }\end{array}$ \\
\hline
\end{tabular}

These qualitative descriptions were implemented by defining values for movement features that could represent the linguistic terms coming from the mentioned qualitative descriptions. The interpretation of the qualitative terms was the following (Angel Fernandez, 2016):

- Anger: 'strong force' was interpreted as TranslationalSpeed = 'High' $(500 \mathrm{~mm} / \mathrm{s})$ speed for 
$50 \mathrm{~mm}$ and then a sudden drop, TranslationalSpeed $=$ 'Normal' $(300 \mathrm{~mm} / \mathrm{s})$. An oscillation during the whole trajectory was also added: BodyRotation $=0.086 \mathrm{rad} ;$ Angular Velocity $=3.5 \mathrm{rad} / \mathrm{s}$.

- Happiness: for the first $50 \mathrm{~mm}$ the velocity was $500 \mathrm{~mm} / \mathrm{s}$, then it went down to $400 \mathrm{~mm} / \mathrm{sec}$ for another $50 \mathrm{~mm}$, and then up again to $800 \mathrm{~mm} / \mathrm{sec}$ for the rest of the movement. An oscillation angle $(\theta)$ between $[0.26,-0.26]$ radians with an angular velocity $(\omega)$ of $3 \mathrm{rad} / \mathrm{s}$ was also added.

- Sadness: the required "low energy" was interpreted as low speed and then slowing down the pace, thus a velocity of $150 \mathrm{~mm} / \mathrm{sec}$ was used for the first $200 \mathrm{~mm}$, a velocity $250 \mathrm{~mm} / \mathrm{sec}$ for the next $200 \mathrm{~mm}$, and for the rest a velocity of $300 \mathrm{~mm} / \mathrm{sec}$. This emotion expression did not include any angular rotation.

- Content: the velocity was $150 \mathrm{~mm} / \mathrm{s}$ for the first $200 \mathrm{~mm}$, then $300 \mathrm{~mm} / \mathrm{s}$ for the rest. The angle of oscillation was $0.175 \mathrm{rad}$ with angular velocity $(\omega)$ of $0.17 \mathrm{rad} / \mathrm{s}$

As it can be seen, having no framework at that time, all the feature values were defined experimentally, and were not framed conceptually.

Subjects were asked to select the emotion label corresponding to the robot's performance they were exposed to, among eight terms, including confounding terms in the same four quadrants of the ArousalValence model as the shown emotions, namely: Frustration, Boredom, Astonishment, and Tiredness. To another set of subjects was asked to write a free term to denote the robot's performance. None of the two results was satisfactory: the fixed list trial obtained a slightly better result, mainly due to the constraint imposed to answers, while, also considering to aggregate similar terms, the free answer trial only demonstrated how diverse could be the terms used by people to label this kind of movements.

From this experience, the too qualitative literature descriptions were left, and a further experiment (Angel and Bonarini, n.d.) was set up by showing to subjects a range of compositions of feature values and collecting the intensity the subjects could associate to each term in a set of emotions (Happiness, Sadness, Fear, and Anger) and two mental states that could be confused with them (Tenderness and Excitement). From this experiment its was expected to identify the set of feature values mostly associated to the emotion terms.

It turned out that Fear was associated to retracting at medium speed with a slight oscillation, Sadness to low to medium speed, and low to medium angular speed, Happiness mainly to oscillation (from moderate to strong) on place, Anger to quite fast speed in the subject's direction, and small oscillation. However, the Kippendorff alpha agreement value was low for almost all emotions, so the obtained 'best' values had still to be considered just as questionable suggestions, from a statistical point of view. This modelling step can be considered as an indirect way to identify values to be adopted to express emotions. With reference to the framework, at this stage it had been possible to define the characteristic points of the fuzzy set distribution.

In the next case study (Angel and Bonarini, n.d.), subjects were exposed to movements coming from the feature combinations best associated to the emotion labels (Anger, Happiness, Sadness, and Fear). They had again to pick one out of eight terms to classify the robot's action. Although a certain trend towards validation of the identified parameters is documented, it is still not supported by enough statistical evidence.

A final experiment was performed (Angel and Bonarini, n.d.), showing an unspoken part (the introduction) of the balcony scene from Shakespeare's Romeo and Juliet, with and without emotional expression. Different emotions are involved in this scene, from Anger, to Fear, to Excitation, expressed in sequence by Romeo, interpreted by MARIOS. A statistically significant part of the 127 subjects preferred the representation with emotional expression, so providing an indirect support to it.

In conclusion, from the the MARIOS experience emerged that a statistically significant identification of the parameters of the emotional expression model is hard to obtain, when not strongly driven (in all the trials done, quite confounding terms where included). However, it is possible to identify emotional expressions that could be appreciated by the audience, which is an indirect confirmation that the process done is meaningful. The final emotional model could be framed in the framework and confirmed for Puppy, the next robot presented.

\subsection{Puppy: how easy}

Puppy is a simple 4-wheeled base mounting an mobile upper part, described in Figure 4. It is $20 \mathrm{~cm}$ long and $25 \mathrm{~cm}$ high, and can run at a maximum speed of about $30 \mathrm{~cm} / \mathrm{sec}$.

The 'Regular' value for TranslationalSpeed was $15 \mathrm{~cm} / \mathrm{sec}$ and the other values were distributed in a way analogous to the last model defined for MARIOS.

One of the Happiness patterns for this robot was defined as a sequence of 'Fast' speed, in the 'Towards' direction, followed by a change of direction to 'Away' after $0.5 \mathrm{sec}$, and a new change to 'Towards' for $0.5 \mathrm{sec}$. This gives an alternate movement of the base, that can be repeated as many times as desired. 


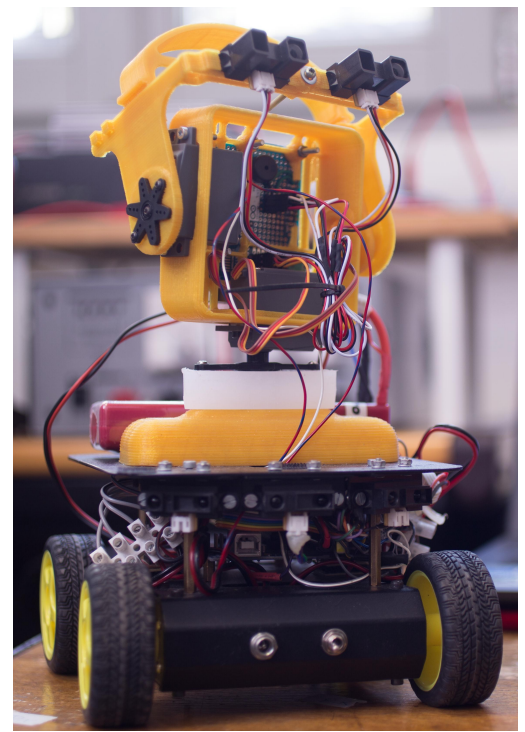

Figure 4. Puppy, a differential drive robot, holding a vertical element that can rotate left and right on its axis, together with an horizontal element rotating up and down on a joint positioned on its center. This implements a disentangled yaw and pitch movement w.r.t. the mobile base.

At the same time, the upper part is moving the horizontal element up and down with the same pace. An alternative implementation of Happiness, is also moving forward and backward, but a component of 'Clockwise' 'SlowRotation' is now added to the forward part, and the same 'CounterClockwise' to the backward part, thus obtaining alternating backward and forward arcs: since the forward part is slightly longer, the resulting path is bringing the robot on, along a kind of lateral zig-zag, while the upper element is turning left and right, and up and down at the same pace.

Sadness was implemented by 'Slow' movements (TranslationalSpeed $=5 \mathrm{~cm} / \mathrm{sec}$ ), backward for the base, functional to move away from the non-human obstacle, alternated 'Clockwise' and 'Counter-clockwise' for the upper part and with the horizontal part that slowly reached a low position.

These emotional expressions were shown both live, without context, to more than 200 people, and in video (available from https://www.youtube.com/ watch?v=pfAedWxrF7c), with the mentioned context, to hundreds, in different occasions, obtaining an extremely good recognition rate (more than $93 \%$, when explicit feedback was collected on 84 people on the live performance, and $96 \%$ of the 151 collected feedbacks on video representation), even at a congress of ethologists, where one of the comments was: "It seems a tiny dog pet", from which, the name 'Puppy' thereafter. No

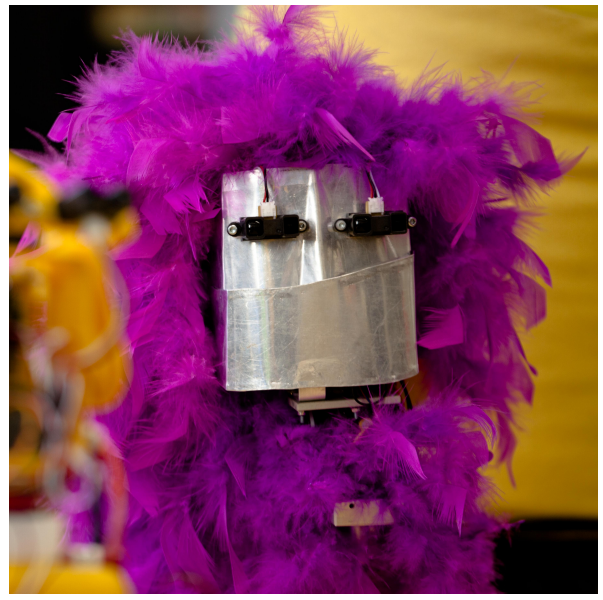

Figure 5. Concierge, in its funky version.

elements specifically inspired to a dog body are present on the robot body, so the connection to a personal emotional experience could be only related to motion.

The implemented emotion expressions were coherent with those implemented in MARIOS, with a lower absolute reference speed, since Puppy is smaller than MARIOS. Puppy has also a movable part on the top, that could exploit a kind of bending, actually, a rotation with respect to a vertical part that could in turn rotate along its axis. These specific movements are not really possible to humans or animals, but the possibility of bending and rotating is enough to give strong cues about emotional expression.

\subsection{Concierge: a time-critical task}

Concierge is a robotic metal box implementing yaw and pitch movements w.r.t. its bottom center (see Figure 5). It is intended to stay on the side of an office door, following people passing in the corridor, and allowing them entering in the office when they get close to the door, or denying access if the office occupant wouldn't be disturbed or is not in the office. This apparently easy task is performed by using two infra-red distance sensors and implementing a simple strategy to track the passing person: when a distance less than the maximum is detected by a sensor, the head moves to detect a similar distance also with the other sensor, thus trying to keep the moving person in the range of both sensors.

The task requires a continuous attention when following the person with sight, and there is not much space for additional expressive movements, since the rotation speed is bounded by functional constraints to the speed of the person passing by. In this case, the emotional expression was aimed at supporting a 
character coherent with the box dressing; we limited it to the reaction speed and the yes/no movements done when the person gets close.

The appearance of this robot is undoubtedly that of a head, although all components (except dressing) are just fully functional to the task: the box is dimensioned to contain electronics and motors, the sensors should be aligned, slightly divergent, a yes/no signal had to be produced when people approaches the door. Here, the emotional model was superimposed to the motion generated in real time to match the functional need of following people at their speed. A 'Fast' movement to get the person in the range of both IR beams turned out in nervous start and stop movement. This is fully compatible with the funky appearance of the implementation reported in Figure 5, and exploits a nice mechanical effect on hair/feathers.

A relatively slower movement is compatible with the wise wizard character suggested by an alternative dressing, where the movement composition led sometimes to annoyed, picky movements to recover missing people lost because of the the too slow movement. It was compatible with the character, and its was left as a compromise between functionality and emotional expression. The same movement characteristics have been implemented for the yes/no actions: jittery for the funky version, slow and calm for the wise old man (movie available at https:// www youtube. $\mathrm{com} /$ watch?v=lcNuKryzmjo).

\subsection{Teo: when emotions are critical}

Teo (Bonarini et al., 2016) (see Figure 6) has been implemented as a robot to interact with children affected by cognitive Developmental Disorder (DD), e.g., Autistic Spectrum Disorder (ASD). In this case, it plays the role of a technological substitute for a person with whom DD children can freely develop a relationship. Among the other functionalities, a small set of emotional movements were implemented, integrated with sound and light effects. The robot base is the same as MARIOS, but the top part is made of soft cloth covering a bunch of soft polystyrene microballs (used also for cushion filling), in turn covering a hard shell.

The top part is soft, and holds touch sensors that enable the distinct perception of hugs, caresses, slaps, and punches. The corresponding emotional movements are implemented as quite small movements, to keep contact with the subject. Reaction to hugs and caresses is just a kind of vibration implemented as small, alternate micro-movements: $0.5 \mathrm{~cm}$ path, fast back and forth movements, coherent with what done for Puppy's Happiness, but more gentle, obtained by

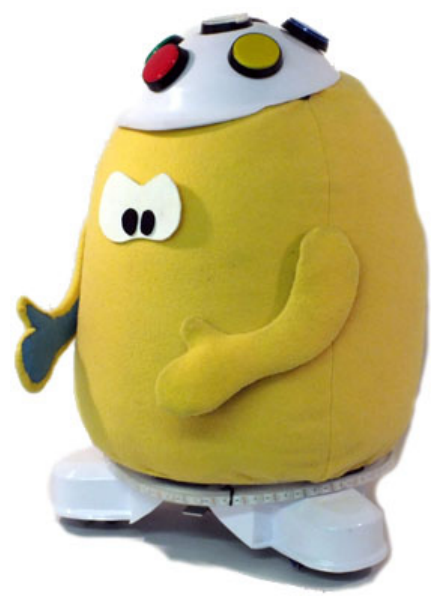

Figure 6. Teo, a holonomic base for ASD children. Eyes, arms, and mouth can be attached by the kids in the familiarization phase, if desired.

reducing the 'Fast' speed classification within the corresponding fuzzy area. Reaction to harsh interaction was implemented, depending on the strength of the input signal, as Fear, moving quickly away from the subject, staying away and vibrating visibly, or as Anger, moving suddenly in the direction of the subject: the path is linear, in the subject's direction, and the pattern includes bursts (rush and stop) and small, fast rotations. These movement descriptions were coherent with those finally implemented in MARIOS.

Teo was appreciated in the centres where it was tested, both by therapists, who could control the activation of the emotional reaction, and subjects, thus positively passing its indirect evaluation. By decision of the therapists, it was not possible to perform any statistical analysis on these experiences, since they know that each ASD person is different from any other, and each of the sessions had to be developed along a path to be tuned in real-time upon reactions of the subject.

\section{Conclusion}

A framework to express emotions in NOBIBots is presented in this paper. The framework is based on the definition of emotional patterns with linguistic terms associated to fuzzy sets defined to classify motion feature values. These patterns can be superimposed to the functional motion of the robot to add emotional expression to task execution. Classifications of the feature values in terms of fuzzy sets can be adapted to robots and contexts, while the same emotional patterns can in principle be shared among different applications. 
The framework has been developed upon experiences concerning the implementation of robots able to show recognizable emotion-based movements; these have been summarized, together with issues that may arise in different situations to apply the framework.

The proposed framework is being applied in the implementation of robotic toys, emotional robotic objects, and robogames (Martinoia et al., 2013) both for normally developed people and for people with special needs. The aim of this research activity is to understand how to support the implementation of emotional expressions to be added to task-oriented movements for today's social robots.

We left for the final paragraphs the answer to the question of the paper's title: "Can my robotic home cleaner be happy?" This answer is not based on possibly questionable experiments, but on reasoning about the issues discussed in this paper. If we assume that a good representation for Happiness requires fast movements, with sudden changes of direction, we may observe that most home cleaners have a limited speed, compatible with their tasks, and there is little space for variability. In particular, speed is designed to be more or less constant, and operational speed (the 'Regular' one) is quite close to the maximum, as mentioned in Section 5. A slow expression of Happiness is not compatible with the usual dimensions of home cleaners, perceived as small and potentially expected to show high acceleration. Happiness might exploit also a movement to the 'high' direction of some part of the body (limbs, chest, or head) and openness, but robotics cleaners do not have any movable part in their body. Thus, we may sadly conclude that they cannot really be happy. This is coherent with the results of (Wiltgen, Beer, McGreggor, Jiang, and Thomaz, 2010), who had to implement the happy expression for Roomba with only sounds and light, and could obtain acceptable recognition rate only by presenting a carefully designed movie to provide a clear context.

In principle, home cleaners could be sad, by adding small rotations to a very slow movement, preferably going backward (but forward motion could also be accepted, to leave them performing their task). Rotations could affect floor coverage, given the dualdrive kinematics of most of these robots, and this has to be managed to reduce the loss of performance (also related to slow speed) due to emotional expression. Moreover, also Sadness would in principle need some closure in the robot body, and the effectiveness of a displacement-only movement should be tested.

In conclusion, the framework turns out to be a tool to frame and formally describe (possibly alternative) models for emotion expression, to share them together with reference to actual values used in robots, to reuse them in other applications and to reason about possibility to express emotion compatible with robot features.

\section{Acknowledgements}

We would like to thank the many students that contributed to the development of the robots mentioned in this paper, in particular Julian Angel who implemented what was called here MARIOS, and the publishers of enlightening YouTube movies. The structures of Concierge, Puppy and Teo have been designed in collaboration with Maximiliano Romero, Design Department, Politecnico di Milano. Teo was developed within the project 'KROG: Kinect and Robot based interactiOn and Gaming for ASD children', funded by Politecnico di Milano within the Polisocial Award Program 2013-2015.

\section{References}

Aldebaran. (2016). Pepper robot. https:// www. aldebaran. com/en/cool-robots/pepper. (Last accessed: April 2016)

Angel, J., and Bonarini, A. (n.d.). Conveying emotion with non-human-like robot platform. In The 25th ieee international symposium on robot and human interactive communication, ro-man 2016 (p. In press).

Angel, J., and Bonarini, A. (2014, Aug). Studying peoples emotional responses to robots movements in a small scene. In The 23rd ieee international symposium on robot and human interactive communication, ro-man 2014 (p. 417-422).

Angel Fernandez, J. M. (2016). Theatrebot (Unpublished doctoral dissertation). Politecnico di Milano, Milano, I.

Atkinson, A. P., Dittrich, W. H., Gemmell, A. J., and Young, A. W. (2004). Emotion perception from dynamic and static body expressions in pointlight and full-light displays. Perception, 33(6), $717-746$.

Barakova, E. I., and Lourens, T. (2010). Expressing and interpreting emotional movements in social games with robots. Personal and ubiquitous computing, $14(5), 457-467$.

Bloom, T., and Friedman, H. (2013). Classifying dogs(canis familiaris) facial expressions from photographs. Behavioural processes, 96, 1-10.

Bonarini, A., Garzotto, F., Gelsomini, M., Romero, M., Clasadonte, F., and Çelebi Yilmaz, A. N. (2016). A huggable, mobile robot for developmental disorder interventions in a multimodal interaction space. In Proceedings of the 
25th ieee international symposium on robot and human interactive communication (ro-man 2016) (p. In press).

Cacioppo, J., Tassinary, L., and Berntson, G. (2000). Handbook of psychophysiology. University Press.

Camurri, A., Lagerlöf, I., and Volpe, G. (2003). Recognizing emotion from dance movement: comparison of spectator recognition and automated techniques. International journal of humancomputer studies, 59(1), 213-225.

Crane, E., and Gross, M. (2013). Effortshape characteristics of emotion-related body movement. Journal of Nonverbal Behavior, 37(2), 91-105. Retrieved from http://dx.doi . org/10.1007/s10919-013-0144-2 doi: 10 $.1007 / \mathrm{s} 10919-013-0144-2$

Csikszentmihalyi, M. (1997). Finding flow: The psychology of engagement with everyday life. Basic Books.

Darwin, C. (1998). The expression of the emotions in man and animals (3rd ed.). London, UK: Harper Collins. (Preface by P. Ekman)

Dittrich, W. H., Troscianko, T., Lea, S. E., and Morgan, D. (1996). Perception of emotion from dynamic point-light displays represented in dance. Perception, 25(6), 727-738.

Ekkekakis, P. (2012). Affect, mood, and emotion. In G. Tenenbaum, R. C. Eklund, and A. Kamata (Eds.), Measurement in sport and exercise psychology (pp. 321-519). USA: Human Kinetics.

Ekman, P. (1999). Basic emotions. In T. Dalgleish and M. Power (Eds.), Handbook of cognition and emotion (pp. 45-60). New York, NY: Wiley.

Ekman, P. (2004). Emotions Revealed : Recognizing Faces and Feelings to Improve Communication and Emotional Life. Owl Books. Paperback.

Hasbro. (2016). Furby toy. http://www.hasbro.com/ en-us/brands/furby. (Last accessed: April 2016)

Johnstone, K. (2012). Impro: Improvisation and the theatre. Routledge.

Kikhia, B., Gomez, M., Jiménez, L. L., Hallberg, J., Karvonen, N., and Synnes, K. (2014). Analyzing body movements within the laban effort framework using a single accelerometer. Sensors, 14(3), 5725-5741.

Kittmann, R., Fröhlich, T., Schäfer, J., Reiser, U., Weißhardt, F., and Haug, A. (2015). Let me introduce myself: I am care-o-bot 4, a gentleman robot. In Mensch und computer 2015-proceedings (pp. 223-232). Oldenbourg, D: De Gruyter.

Kleinsmith, A., and Bianchi-Berthouze, N. (2013).
Affective body expression perception and recognition: A survey. IEEE Transactions on Affective Computing, 4(1), 15-33. doi: http://doi.ieeecomputersociety.org/10.1109/ T-AFFC.2012.16

Knight, H., and Simmons, R. (2014, Aug). Expressive motion with $\mathrm{x}, \mathrm{y}$ and theta: Laban effort features for mobile robots. In Robot and human interactive communication, 2014 ro-man: The 23rd ieee international symposium on (pp. 267273). IEEE Press. doi: 10.1109/ROMAN.2014 .6926264

Kowalczuk, Z., and Czubenko, M. (2016). Computational approaches to modeling artificial emotionan overview of the proposed solutions. Frontiers in Robotics and AI, 3, 21.

Kozima, H., Michalowski, M. P., and Nakagawa, C. (2009). Keepon. International Journal of Social Robotics, 1 (1), 3-18.

Kuhl, P. K. (1991). Human adults and human infants show a perceptual magnet effect for the prototypes of speech categories, monkeys do not. Perception \& psychophysics, 50(2), 93-107.

Laban, R., and Ullmann, L. (1968). Modern educational dance (2d ed., rev. by Lisa Ullmann. ed.). Praeger New York.

Lakatos, G., Gácsi, M., Konok, V., Brúder, I., Bereczky, B., Korondi, P., and Miklósi, Á. (2014). Emotion attribution to a non-humanoid robot in different social situations. PloS one, 9(12), e114207.

Lang, P. J., Bradley, M. M., and Cuthbert, B. N. (2008). International affective picture system (iaps): Affective ratings of pictures and instruction manual. Technical report A-8.

Lasseter, J. (1987). Principles of traditional animation applied to $3 \mathrm{~d}$ computer animation. $A C M$ Siggraph Computer Graphics, 21(4), 35-44.

Lecoq, J. (2000). The moving body (le corps poetique): Teaching creative theatre. London, UK: Methuen.

Loman, S., and Merman, H. (1996). The kmp: A tool for dance/movement therapy. American Journal of Dance Therapy, 18(1), 29-52.

Lourens, T., Van Berkel, R., and Barakova, E. (2010). Communicating emotions and mental states to robots in a real time parallel framework using laban movement analysis. Robotics and Autonomous Systems, 58(12), 1256-1265.

Machajdik, J., and Hanbury, A. (2010). Affective image classification using features inspired by psychology and art theory. In Proceedings of the 
international conference on multimedia (pp. 83$92)$.

Marsella, S., Gratch, J., and Petta, P. (2010). A blueprint for affective computing. In (p. 21-46). Oxford University Press.

Martinoia, D., Calandriello, D., and Bonarini, A. (2013). Physically interactive robogames: Definition and design guidelines. Robotics and Autonomous Systems, 61(8), 739-748.

Masuda, M., and Kato, S. (2010). Motion rendering system for emotion expression of human form robots based on laban movement analysis. In Ro-man, 2010 ieee (pp. 324-329).

Mentis, H. M., and Johansson, C. (2013). Seeing movement qualities. In Proceedings of the sigchi conference on human factors in computing systems (pp. 3375-3384). New York, NY, USA: ACM.

Moore, R. K. (2012). A bayesian explanation of the uncanny valleyeffect and related psychological phenomena. Scientific reports, 2, 864.

Mori, M. (1970). Uncanny valley. Energy, 7(4), 33-35.

Novikova, J., and Watts, L. (2015). Towards artificial emotions to assist social coordination in hri. International Journal of Social Robotics, 7(1), 77-88. Retrieved from http://dx.doi.org/10 .1007/s12369-014-0254-y doi: $10.1007 / \mathrm{s} 12369$ $-014-0254-\mathrm{y}$

Osgood, C. E., Suci, G., and Tannenbaum, P. (1957). The measurement of meaning. Urbana, IL: University of Illinois Press.

Parlitz, C., Hägele, M., Klein, P., Seifert, J., and Dautenhahn, K. (2008). Care-o-bot 3rationale for human-robot interaction design. In Proceedings of 39th international symposium on robotics (isr), seul, korea (pp. 275-280).

Russell, J. A. (1980). A circumplex model of affect. Journal of Personality and Social Psychology, 39, 1161-1178.

Scherer, K. R. (2010). A blueprint for affective computing. In (p. 21-46). Oxford University Press.

Sony. (2008). Rolly, operating instructions. http:// pdf.crse.com/manuals/3870834321.pdf.

Stanislavski, K. (1989). An actor prepares. London, UK: Bloomsbury.

Thomas, F., and Johnston, O. (1995). The illusion of life: Disney animation. New York, NY: Hyperion.

Wang, H. L., and Cheong, L.-F. (2006). Affective understanding in film. Circuits and Systems for Video Technology, IEEE Transactions on, 16(6), 689-704.
Wiltgen, B., Beer, J. M., McGreggor, K., Jiang, K., and Thomaz, A. (2010). The interplay of context and emotion for non-anthropomorphic robots. In Ro-man, 2010 ieee (pp. 658-663).

Wistort, R. (2010). Only robots on the inside. Interactions, 17(2), 72-74.

Zadeh, L. A. (1965). Fuzzy sets. Information and control, 8(3), 338-353.

Zadeh, L. A. (1975). The concept of a linguistic variable and its application to approximate reasoningi. Information sciences, 8(3), 199-249. 